Article

\title{
Multi-Wavelength Intra-Day Variability and Quasi-Periodic Oscillation in Blazars
}

\author{
Alok C. Gupta \\ Aryabhatta Research Institute of Observational Sciences (ARIES), Manora Peak, Nainital 263002, India; \\ acgupta30@gmail.com
}

Received: 2 December 2017; Accepted: 19 December 2017 ; Published: 25 December 2017

\begin{abstract}
We reviewed multi-wavelength blazars variability and detection of quasi-periodic oscillations on intra-day timescales. The variability timescale from a few minutes to up to less than a days is commonly known as intra-day variability. These fast variations are extremely useful to constrain the size of the emitting region, black hole mass estimation, etc. It is noticed that in general, blazars show intra-day variability in the complete electromagnetic spectrum. However, some classes of blazars either do not show or show very little intra-day variability in a specific band of the electromagnetic spectrum. Blazars show rarely quasi-periodic oscillations in time series data in optical and X-ray bands. Other properties and emission mechanisms of blazars are also briefly discussed.
\end{abstract}

Keywords: active galaxies; BL Lacertae object: BL Lac; quasars: flat spectrum radio quasars; jets, accretion disk

\section{Introduction}

It is commonly accepted that super massive black holes (SMBHs, with masses between $10^{6}$ and $\left.10^{10} \mathrm{M}_{\odot}[1]\right)$ are present in the nuclei of all galaxies with stellar bulges. At any given time, a few percent of these SMBHs are fed with a sufficient amount of gas that they will possess significant accretion discs. The emission of these discs is comparable to the total emission of stars in the entire host galaxy because of a very high efficiency for the conversion of matter into radiation as it spirals into a BH. This is the fundamental mechanism underlying the "active galactic nucleus" or AGN.

Roughly $85-90 \%$ of AGNs have very little radio emission $\left(F_{5 \mathrm{GHz}} / F_{B} \leq 10\right.$, here $F_{5 \mathrm{GHz}}=$ flux at radio $5 \mathrm{GHz}$ and $F_{B}=$ flux at optical $B$ band $4400 \AA$ ) and are therefore called radio-quiet AGNs (RQAGNs). The remaining 10-15\% of AGNs are radio-loud AGNs (RLAGNs). It has been proposed that different types of AGNs can be explained by the idea that different line of sight (LOS) angles can play an important role in understanding their different properties $[2,3]$.

Blazars belongs to the RLAGN class, and their LOS is pointed close to the observer. Blazars show rapid variability at almost all wavelengths of the electromagnetic (EM) spectrum with the emission being strongly polarized (optical linear polarization $\geq 3 \%$ ). Due to their strong and large amplitude variability nature in the complete EM spectrum, they are considered as transient astronomical objects. Blazars include two sub-classes: BL Lacertae objects (BLLs) and flat spectrum radio quasars (FSRQs). BLLs show featureless optical continua (no prominent emission or absorption lines), while FSRQs show prominent emission lines in their optical spectra. The radiation from blazars is dominated by non-thermal emission at all wavelengths, consisting of two broad spectral bumps [4,5]: a low-frequency component from radio to the UV or X-rays, generally agreed to be due to synchrotron radiation from relativistic electrons in the jet, and a high-frequency component from $\mathrm{X}$-rays to $\gamma$-rays, which can be either due to inverse Compton scattering of lower-frequency radiation by the same relativistic electrons (leptonic models i.e., [6]) or due to interactions of ultra-relativistic protons in the jet (hadronic 
models), either via proton synchrotron radiation [7] or via secondary emission from the photo-pion and photo-pair production process ([8] and the references therein).

Blazars can be classified into three sub-classes, depending on the peak frequency of their synchrotron emission: LSPs (low-synchrotron-peaked blazars), consisting predominantly of LBLs (red or low energy or radio selected blazars) and defined by a peak of their synchrotron component at $v_{s y}<10^{14} \mathrm{~Hz}$, ISPs (intermediate-synchrotron-peaked blazars) or IBLs, consisting mostly of intermediate blazars, defined by $10^{14} \mathrm{~Hz}<v_{s y}<10^{15} \mathrm{~Hz}$, and HSPs (high-synchrotron-peaked blazars), all of which are HBLs (blue or high energy or X-ray selected blazars) and which are defined through $v_{s y}>10^{15} \mathrm{~Hz}$ [9]. The high-energy component of the spectral energy distribution (SED) of blazars extends up to $\gamma$-rays, peaking at $\mathrm{GeV}$ energies in LSPs and at $\mathrm{TeV}$ energies in HSPs. Blazar properties are consistent with relativistic beaming, i.e., bulk relativistic motion of the jet plasma at small angles to the line of sight, which gives rise to a strong amplification and rapid variability in the observer's frame.

The study of variability is one of the most powerful tools for understanding the nature and processes occurring in blazars. The variability of blazars can be broadly divided into three classes. Significant variations in flux may occur over a few tens of minutes to the course of less than a day, often called micro-variability, intra-night variability or intra-day variability (IDV) [10]. Short-term variability (STV) can range time scales from days to a few months, and long-term variability (LTV) can have time scales of several months to several years [11].

The variability of observations on IDV timescales in AGN is the most puzzling. The variability of blazars on IDV timescales can provide important clues to the physics of the innermost nuclear regions in these objects. Blazar properties are consistent with relativistic beaming caused by bulk relativistic motion of the jet plasma at small angles to the line of sight, which gives rise to a strong amplification and rapid variability in the observer's frame. Simultaneous multi-wavelength observations of blazars in the entire EM spectrum are an important tool to test several possible models for IDV: shock-in-jet models, accretion-disk-based models, models based on plasma instabilities in shear layers, etc.

\section{Intra-Day Variability in Different EM Bands}

There have been several dedicated monitoring campaigns in which the IDV of blazars has been studied over the entire EM spectrum (e.g., [12-43]; and the references therein).

There are several methods that can be used to find the genuine IDV and variability parameters in time series data. These methods are described by different groups, and relevant source references are cited in their papers. [44] introduced the C-test, which is used by several groups (e.g., [23,30,38,45,46]; and the references therein). However, later, [47] explained that the C-test statistic is too conservative of an approach for IDV detection. IDV detection results can be tested using the F-test, which is a distributed statistic [47]. The F-test is also used by several groups (e.g., [30,38]; and the references therein). A better method called the power enhanced F-test was introduced by $[48,49]$. This method takes care of large brightness differences in a blazar and comparison/standard stars or large brightness differences in comparison/standard stars. It was used by [32]. The $\chi^{2}$-test (as explained by: [30,38] also provides evidence of the presence of IDV in time series data. The analysis of variance (ANOVA) test is a very robust test to investigate the variability in the light curves (LCs) (e.g., [27,30,38,50]; and the references therein). Percentage amplitude variation in the time series data was introduced by [18] and used in several papers (e.g., [23,27,30,38]; and the references therein). Another method called excess variance and fractional rms variability amplitude were introduced by [51,52] and extensively used for getting the variability in AGN LCs (e.g., [37,42,53,54]; and the references therein).

IDV in blazars can be intrinsic to the source or due to extrinsic origin. Interstellar scintillation and gravitational microlensing are the main extrinsic causes of IDV. Interstellar scintillation is only relevant in low-frequency radio observations. Gravitational microlensing is only applicable in a few blazars, which are lensed systems, e.g., the blazar $\mathrm{AO} 0235+135$ at $\mathrm{z}=0.94$ has revealed foreground-absorbing systems at $z=0.524$ and $z=0.851[55,56]$. In the blazars where IDV is detected, low-frequency radio 
observations or gravitationally-lensed sources can also have some intrinsic origin; to find that, we need to observe simultaneous multi-wavelengths of the blazar, and co-related variability in different EM bands will be helpful to find the nature of the variability.

IDV in the high state (pre/post the outburst state) of blazars can be explained by jet-based models, e.g., helical instabilities in the jet $[57,58]$ or turbulence behind the shock in the jet [59]. Jet-based models can explain IDV over the entire range of EM wavelengths. Other theoretical models seek to explain IDV in blazars (mainly in their low-state) involving accretion-disk-based models. These models include pulsations of the gravitational modes of the gaseous disk $[60,61]$ or orbital signatures from "hot-spots" in the gas surrounding the black hole, either from the disk itself or the corona above it [62,63]. Accretion-disk-based models can explain the variations in optical, UV and X-ray bands, but are difficult to connect to the observed rapid variability in $\gamma$-rays. Plasma instabilities in the jet (e.g., Kelvin-Helmholtz-type instabilities due to the interaction of a fast inner spine of the jet with a slower, outer layer) could play an important role in the production of IDV at a variety of wavelengths [64].

\subsection{IDV in Gamma-Rays}

Most of the ground- and space-based gamma-ray experiments are not sensitive enough to observe blazars with a few minutes time resolution. Therefore, it is extremely difficult to do IDV studies of blazars. However, there is an excellent IDV observation of the blazar PKS 2155-304 in an exceptionally very high energy gamma-ray flare observed on 28 July 2006 using HESS (High Energy Stereoscopic System). The IDV LC obtained with a time resolution of $1 \mathrm{~min}$ and IDV is seen down to the 10-min scale, which gives a Doppler factor of more than 100 . The average flux at $>200 \mathrm{GeV}$ during outburst was $\sim 7$-times the flux observed from the Crab Nebula [22]. The LAT (Large Area Telescope) on board the Fermi gamma-ray space telescope (Fermi-LAT; [65]) has been observing the sky in the gamma-ray spectrum since its launch in June 2008. Fermi-LAT covers $20 \mathrm{MeV}-300 \mathrm{GeV}$ energies and made a revolution in the discovery of the gamma-ray emission of blazars. It has observed several blazars in flaring state and detected strong IDV ([33-36,41,66-68]; and the references therein). Fermi-LAT LCs with a time resolution of minutes of blazars were presented by [33,41], while [34-36,66-68] presented Fermi-LAT LCs of blazars with a few hours time bin. [33] searched for IDV in three FSRQs, 3C 454.3, 3C 273 and PKS B1222+216, in the energy E $>100 \mathrm{MeV}$ and set the upper limit on the observed characteristic time scales, which were of the order of $<2-3 \mathrm{~h}$, which suggests that the location of the $\gamma$-ray emission region could be within the broad-line region (BLR). Based on the 3.75 years of data of the FSRQ PKS 1510-089 in the energy range 0.1-300 GeV, [66] reported the doubling time in flare rise stage as $1.3 \pm 0.12 \mathrm{~h}$, while $1.21 \pm 0.15 \mathrm{~h}$ during flare decay. [34] reported Fermi-LAT LC of the blazar PKS 1222+216 with a 6-h time bin showing the asymmetric rise profiles, but rapid decline during the April 2010 flare. Paliya et al. (2015) reported the giant $\gamma$-ray outburst observed in the FSRQ 3C 279 in March-April 2014 using Fermi-LAT. They reported $\gamma$-ray flux $\left(1.21 \pm 0.10 \mathrm{ph} \mathrm{cm}^{-2} \mathrm{~s}^{-1}\right.$ in the energy range $0.1-300 \mathrm{GeV}$ and flux doubling time as $1.19 \pm 0.36 \mathrm{~h}$. [41] reported $\gamma$-ray IDV in 3C 279 , which showed a giant outburst on 16 June 2015 with a peak flux of $\sim 3.6 \times 10^{-5} \mathrm{ph} \mathrm{cm}^{-2} \mathrm{~s}^{-1}$ at $>100 \mathrm{MeV}$ and flux doubling times of less than $5 \mathrm{~min}$. The same flare in 3C 279 on 16 June 2015 was also studied by Paliya 2015 with the observations taken for 16 days (four days before the flare started and six days after the flare ended), and the shortest doubling time was measured as $2.2 \pm 0.3 \mathrm{~h}$ with $\sim 9 \sigma$ significance, from $\gamma \gamma$ pair opacity arguments; the minimum Doppler factor is reported to be 14; and the location of the $\gamma$-ray emitting region is claimed to be either at the outer edge of BLR or farther out from it. [67] reported a twin $\gamma$-ray flare in 3C 279 in December 2013, which had similar intensity as the flare in April 2014 [35], but less intense than the April 2015 flare. The $\gamma$-ray flux doubling time of $3.04 \pm 0.77 \mathrm{~h}$ was detected. [68] studied $\gamma$-ray flares of 3C 279 observed during December 2013-April 2014. Results reported in [68] and [67] are in good agreement. 


\subsection{IDV in X-Rays}

A pilot project on searching for IDV in blazars was initiated ([28,37,42,43,54]; and the references therein). A sample of four HBLs observed on 23 occasions by XMM-Newton was studied; IDV timescales ranging from 15.7-46.8 ks were found on eight occasions; in 13 cases, IDV timescales were longer than the data length, and the hint of weak quasi periodic oscillations (QPOs) was observed on one LC each of blazars ON 231 and PKS 2155-304 [28]. Reference [69] took the sample of 104 nearby AGN $(\mathrm{z}<0.4)$ observed with XMM-Newton. The AGN sample also includes several blazars. They did power-spectrum density (PSD) analysis of all those LCs and reported IDV variability parameters. The LBL 3C 273 observed on 24 occasions during 2000-2012 with XMM-Newton and on IDV timescales have shown occasionally small amplitude variability [37]. A complete sample of 12 LBL and IBL with 50 IDV LCs taken with XMM-Newton was compiled [37,54]. It is noticed that the duty cycle of genuine IDV detection in LBL and IBL in the X-ray band is only $4 \%$ (two out of 50 LCs) [54]. It is concluded that probably the peak of the spectral energy distribution seems to be responsible for the IDV properties [54]. In a recent study, [42] reported the search for X-ray IDV in five TeV blazars using NuStar . Four TeV blazars have shown large amplitude IDV, using the auto-correlation function (ACF). The IDV timescale in the range of 2.5-32.8 ks was reported in eight LCs of Mrk 421; a timescale of about $8.0 \mathrm{ks}$ for one LC in Mrk 501; and timescales of 29.6-57.4 ks in two LCs of PKS 2155-304. In general, soft (3-10 keV) and hard (10-79 keV) LCs were well correlated, which indicated that the same population was emitting soft and hard X-rays. IDV timescales are used to calculate $\delta$ the Doppler factor, $B$ the magnetic field, $\gamma$ the Lorentz factor and $R$ the size of the emitting region (see Table 4, [42]. Recently, in another search for X-ray IDV, 83 LCs of the TeV blazar Mrk 421 taken during 1999-2015 with Chandra were studied [43]. IDV timescales ranging $2.4-30.0 \mathrm{ks}$, IDV duty cycle $\sim 77 \%$, soft $(0.3-2.0 \mathrm{keV})$ and hard $(2.0-10.0 \mathrm{keV})$ LCs well correlated with zero lag were found. IDV timescales were also used to calculate $\delta$ the Doppler factor, $B$ the magnetic field, $\gamma$ the Lorentz factor and $R$ the size of emitting region for the blazar Mrk 421 [43]. For Mrk 421, we found that shortest IDV timescale $\approx 2.4 \mathrm{ks}, \delta=25, B>0.26 v_{18}^{-1 / 3} \mathrm{G}$, where $v_{18}=$ critical synchrotron emission frequency in terms of $10^{18} \mathrm{~Hz}$ for the Chandra satellite, electron Lorentz factor $\gamma \geq 1.92 \times 10^{5} v_{18}^{2 / 3}$ and the radius of the emitting region $\mathrm{R} \leq 1.7 \times 10^{15} \mathrm{~cm} \mathrm{[43]}$.

\subsection{IDV in Optical and Infrared Bands}

The first evidence of optical micro-variability was reported in BL Lacertae by [12]. The result of this paper motivated several groups around the globe to start dedicated projects to search for optical micro-variability in blazars. Optical IDV in blazars was pioneered by the U.S. group in which they studied optical micro-variability in five blazars (e.g., [12-16]) and reported that the probability of finding genuine micro-variability is about $80 \%$ for the blazar continuously monitored for $<8 \mathrm{~h}$. An extensive search for optical IDV in a sample of 34 BLLs from the 1 Jy catalog was done by [18]. IDV was detected in 28 out of 34 BLLs ( $82 \%)$, and $75 \%$ of the variable BLLs changed significantly over a time span $<6 \mathrm{~h}$. However, these data lack continuity in the LCs. [70] compiled the optical IDV studies of blazars till $\sim 2004$ and noticed that the occurrence of IDV of blazars if observed for less than $6 \mathrm{~h}$ was about $60-65 \%$. If the blazar were observed for more than $6 \mathrm{~h}$, then the possibility of IDV detection was about $80-85 \%$. Several hundred nights of optical IDV searching in blazars were done by different Chinese groups (e.g., [71-88]; and the references therein). Significant IDV was detected in most of these observations, but in several papers, the data lack continuity in the LCs. Indian groups also performed extensive searches for optical/IR IDV in blazars with collaborators around the globe (e.g., [11,20,23,24,26,27,29-32,38-40,89-94]; and the references therein). There are some other collaborations world wide that are performing searches for optical/NIR IDV of blazars (e.g., [19,95-109]; and the references therein). The important results reported in these papers are: (i) LBLs and IBLs show large amplitude IDV with high duty cycles; (ii) HBLs either do not show IDV or if they do, the amplitude of variability and the duty cycle of IDV detection are less compared to LBLs and IBLs; (iii) cross-correlation analysis in different optical bands on IDV timescales in general shows strong correlation with zero lag, which implies that the emissions in different optical bands are 
co-spatial, but occasionally, a few minute time lags are also reported; (iv) optical color variation on IDV timescales shows a range of properties, e.g.: sometimes, no color variation is seen; on some occasions, BLLs are bluer when brighter (BWB) and FSRQs are redder when brighter trend (RWB); and rarely, the opposite trend is also noticed; (v) IR/optical SED in general fits to a power-law, but occasionally shows a big blue bump (BBB), which shows the signature of thermal emission from the accretion disk; (vi) IDV LCs are used to get a variable timescale, which gives a clue about the size of the emitting region, black hole mass estimation of the blazar; etc. [110].

\subsection{IDV in Radio Bands}

IDV in radio bands are a mixture of intrinsic and extrinsic origins. Mostly, observations at centimeter and meter wavelengths have dominant extrinsic variability, which is due to interstellar scintillation of radio waves caused by the turbulent interstellar medium of the Milky Way, while IDV are in the millimeter wavelength of the intrinsic origin. Radio IDV studies of blazars were pioneered by the 100-m Effelsberg radio telescope in Germany, as well as other radio and $\mathrm{mm}$ wavelength telescopes (e.g., [17,25,102,111-121]; and the references therein).

Reference [111] did simultaneous optical and radio monitoring of blazars. Reference [17] reported for the first time the correlated optical and radio IDV in the blazar S5 0716+714. To test the inverse-Compton (IC) catastrophe scenario in the blazar S5 0716+714, extensive observational campaign in the radio and $\mathrm{mm}$ wavelengths were coordinated [102,112,115], and the lower limits to the brightness temperature were derived from the inter-day variations exceeding the $10^{12} \mathrm{~K}$ IC-limit by up to 2-4 orders of magnitude. Reference [113] reported radio IDV of the blazar J 1128+5925 in three frequencies, i.e., 2.7- and 10.45-GHz observations using the 100-m Effelsberg radio telescope in Germany, and 4.8-GHz observations using the 25-m radio telescope in Urumqi, China. The observed frequency-dependent IDV in the source was in good agreement with the prediction from interstellar scintillation. VLBA observation of the blazar J1128+592 was reported by [114], and with VLBA observations, they detected an east-west-oriented core-jet structure with no significant motion in its jet. Radio IDV in blazars was studied where the variability characteristics have changed abruptly by interstellar scintillation [116,117]. Radio IDV at $4.8 \mathrm{GHz}$ using the 25-m Urumqi, China telescope for the blazars S5 $0716+714$ and 1156+295 was reported by [118,119]. Simultaneous IDV in the X-ray, four optical bands and three frequencies in the radio spectrum were reported by [25]. IDV was detected in all three radio frequencies, and it was also noticed that low and high frequency correlations do not peak at zero lag, which shows that low frequency radio observation is the combined effect of an intrinsic and an extrinsic mechanism. Optical and radio IDV observations were carried out by [121]. IDV observation along with VLBI analysis was carried out for the blazar S4 0917+624 [120].

\section{Quasi Periodic Oscillations in Intra-Day Time Series Multi-Wavelength Data}

Detection of quasi-periodic oscillations (QPOs) in time series data are very rare in AGN. In the last decade, several detections of QPOs in AGN on diverse timescales ranging from as short as a few minutes and as long as a few years using $\gamma$-ray, X-ray, optical and radio time series data were made (e.g., [122-139]; and the references therein). However, only a few claims of QPO detection on IDV timescales using X-ray and optical monitoring data of a few blazars have been reported [123-125,127].

There are several methods that can be used to search for QPO or periodic signals in time series data. These methods are described by different groups, and relevant source references are cited in their papers. Reference [122] used power spectral density (PSD) and data folding. Reference [123] used wavelet analysis, whereas [124] used wavelet plus randomization technique. Weighted wavelet z-transform (WWZ) and the Lomb-Scargle periodogram (LSP) were used by [139]. Multiple analysis techniques, e.g., structure function (SF), wavelet analysis, data folding, PSD and multi-harmonic AoV periodogram (mhAoV), were used by [125]. SF and the auto-correlation function (ACF) were used to get the variable timescale and QPOs [28,42]. One or multiple methods used in these papers were used in other searches for QPOs. 
QPOs in blazars on IDV timescales can be explained by several standard models of AGN. One of the simplest models by which the central BHs of AGN would have the QPOs attributed can be explained by the presence of a single dominating hot-spot on the accretion disk (e.g., [63,140]. Using $\mathrm{QPO}$ or a nearly periodic signal, the period can be used to estimate the $\mathrm{BH}$ mass for non-rotating (Schwarzschild) BH and maximally-rotating (Kerr) BH. The detailed explanation is given in [124]. Other alternative possible mechanisms for QPOs in blazars on IDV timescales can also have a disk origin or can arise from relativistic jets. The former class includes small epicyclic deviations in both radial and vertical directions from exact planar motions within a thin accretion disk (e.g., [141]) and trapped pulsational modes within a disk (e.g., [123,142]). Using the detailed explanation of [142], one can also get the BH mass of the blazar. There are various jet models that also can explain the QPO detection in blazars on IDV timescales, e.g., a shock propagating down a jet in which the jet structure is quasi-helical and changes in electron density or magnetic field can produce QPO, and a short-lived QPO can be due to turbulence behind the shock in the relativistic jet (e.g., $[59,143,144])$.

\subsection{In the Optical Band}

Reference [124] selected 20 optical IDV light curves of the blazar S5 0716+714 from a database of 102 lights curves taken in a three-year span [21]. They used wavelet analysis along with the randomization test and found strong evidence for nearly periodic variations on five light curves with a probability $>99 \%$. The period for these five light curves was found in the range of $25 \mathrm{~min}-73 \mathrm{~min}$, which led to $\mathrm{BH}$ mass ranging 2.47-7.35 $\times 10^{6} \mathrm{M}_{\odot}$ and 1.57-4.67 $\times 10^{7} \mathrm{M}_{\odot}$ for non-rotating $\mathrm{BH}$ and maximally-rotating $\mathrm{BH}$, respectively. Another piece of evidence of QPO detection in the optical band on the same blazar S5 0716+714 was reported by [127]. They found a QPO period of $\sim 15$ min using various techniques (e.g., SF, LSP, PSD, data folding). This period yields the BH mass $1.5 \times 10^{6} \mathrm{M}_{\odot}$ and $9.6 \times 10^{6} \mathrm{M}_{\odot}$ for non-rotating $\mathrm{BH}$ and maximally-rotating $\mathrm{BH}$, respectively.

\subsection{In X-Rays}

Using the wavelet technique, Reference [123] analyzed 19 observations of 10 AGN observed with the EPIC/pn detector on board XMM-Newton and detected a QPO period of $3.3 \mathrm{ks}$ in one light curve of the blazar 3C 273. The QPO period is used to get the black hole (BH) mass of the blazar. They estimated the $\mathrm{BH}$ mass of the blazar to be $7.3 \times 10^{6} \mathrm{M}_{\odot}$ and $8.1 \times 10^{7} \mathrm{M}_{\odot}$ for non-rotating $\mathrm{BH}$ and maximally-rotating $\mathrm{BH}$, respectively. In another observation of EPIC/pn of XMM-Newton for the blazar PKS 2155-304, Reference [125] detected a QPO period of $4.6 \mathrm{~h}$ that was present for $\sim 3.8$ cycles. This QPO detection was verified by various techniques (e.g., SF, PSD, MHAoV, data folding and wavelet). The BH mass of the blazar was estimated to be $3.29 \times 10^{7} \mathrm{M}_{\odot}$ and $2.09 \times 10^{8} \mathrm{M}_{\odot}$ for non-rotating $\mathrm{BH}$ and maximally-rotating $\mathrm{BH}$, respectively.

\section{Conclusions}

With the extensive studies of IDV in the radio to optical bands in approximately the last three decades and in high energies (X-ray and $\gamma$-rays) in the last decade, we reach the following conclusions:

- Blazars show large amplitude IDV in radio bands, which is basically the mixture of extrinsic and intrinsic mechanisms.

- $\quad$ LBLs and IBLs show large amplitude IDV in optical/IR bands with a high duty cycle.

- HBLs either do not show optical/IR IDV or if they do, the amplitude is low and the duty cycle is very small compared to LBLs/IBLs.

- In general, blazars do not show color variation on IDV timescales; however, occasionally this is seen.

- Optical inter-band cross-correlation shows that in general, there is no time lag in different optical bands. On some occasions, the time lag of a few minutes is reported in different optical bands.

- Optical/IR SEDs are well fitted to a single power law. 
- Sometimes optical/IR SEDs show a big blue bump, which is a signature of emission from the accretion disk.

- In X-rays, HBLs show large amplitude IDV, and the duty cycle is high.

- In X-rays, LBLs/IBLs either do not show IDV or if they do, the amplitude is less. The duty cycle of X-ray IDV for LBLs/IBLs is much less compared to the duty cycles for HBLs.

- In general, hard and soft X-ray LCs of blazars show strong cross-correlation with zero lag, which implies that the emissions in the hard and soft bands are co-spatial.

- IDV timescales in different EM bands are used to get the black hole mass of the blazars, the size of the emitting region and the Doppler factor $\delta$.

- In general, the sensitivity of very high energy $\gamma$-ray facilities is poor, but occasionally, blazars are observed on time resolutions of a few minutes to a few hours. The best time resolution $\gamma$-ray light curves give a high Doppler factor of $\sim 100$ for the blazar PKS 2155-304.

- QPOs in blazars on IDV timescales are rare.

- Occasionally, QPOs on IDV timescales are detected in a few blazars in the X-ray and optical bands.

\section{Future Projects}

In the last three decades, there have been several attempts to search for IDV in blazars, but still, we need many new observations in the complete EM spectrum to better understand the IDV properties of various sub-classes of blazars and detected QPOs in blazars. Below, I list a few projects that I think need focused effort to search for IDV and QPOs in blazars.

- Simultaneous low and high frequency radio IDV should be studied with other EM bands with almost a similar time resolution. This will help us to separate out the extrinsic component of low-frequency IDV, which is responsible for the high brightness temperature. For such observations, instead of using single-dish radio telescopes, interferometric telescopes will be a much better option.

- For a large sample of blazars, IDV studies in IR bands are not yet performed with focused effort, which needs to be done.

- With the help of the Fermi-LAT and VHE gamma-ray facilities, a large number of new HBLs has been discovered. Extensive IDV of HBLs in the optical bands should be done to see if the IDV duty cycle is really much less for HBLs in optical bands in comparison to LBLs and IBLs.

- Extensive IDV studies for LBLs and IBLs are not yet done in X-rays and $\gamma$-rays, which can be done to see if the IDV properties in these bands for LBLs and IBLs are similar to HBLs.

- Still, there are only a few pieces of evidence when blazars are observed in $\gamma$-rays on IDV timescales. Upcoming CTA can contribute to this project.

- QPO is rarely detected in blazars on diverse timescales in different EM bands. It is even much rarer to detect QPO in blazars on IDV timescales. A focused effort with high time resolution and good S/N data is required to search for QPOs on IDV timescales in various EM bands.

Author Contributions: The author was asked by the journal to write the review article. The author planned and wrote the article.

Conflicts of Interest: The author declares no conflict of interest.

\section{References}

1. Woo, J.-H.; Urry, C.M. Active Galactic Nucleus Black Hole Masses and Bolometric Luminosities. Astrophys. J. 2002, 579, 530-544.

2. Antonucci, R. Unified models for active galactic nuclei and quasars. Annu. Rev. Astron. Astrophys. 1993, 31, 473-521.

3. Urry, C.M.; Padovani, P. Unified Schemes for Radio-Loud Active Galactic Nuclei. Pub. Astron. Soc. Pac. 1995, $107,803-845$. 
4. Ghisellini, G.; Villata, M.; Raiteri, C.M.; Bosio, S.; de Francesco, G.; Latini, G.; Maesano, M.; Massaro, E.; Montagni, F.; Nesci, R.; et al. Optical-IUE observations of the gamma-ray loud BL Lacertae object S5 0716+714: Data and interpretation. Astron. Astrophys. 1997, 327, 61-71.

5. Fossati, G.; Maraschi, L.; Celotti, A.; Comastri, A.; Ghisellini, G. A unifying view of the spectral energy distributions of blazars. Mon. Not. R. Astron. Soc. 1998, 299, 433-448.

6. Krawczynski, H. TeV blazars-Observations and models. New Astron. Rev. 2004, 48, 367-373.

7. Mücke, A.; Protheroe, R.J.; Engel, R.; Rachen, J.P.; Stanev, T. BL Lac objects in the synchrotron proton blazar model. Astropart. Phys. 2003, 18, 593-613.

8. Böttcher, M. Modeling the emission processes in blazars. Astrophys. Space Sci. 2007, 309, 95-104.

9. Abdo, A.A.; Ackermann, M.; Agudo, I.; Ajello, M.; Aller, H.D.; Aller, M.F.; Angelakis, E.; Arkharov, A.A.; Axelsson, M.; Bach, U.; et al. The Spectral Energy Distribution of Fermi Bright Blazars. Astrophys. J. 2010, $716,30-70$.

10. Wagner, S.J.; Witzel, A. Intraday Variability In Quasars and BL Lac Objects. Annu. Rev. Astron. Astrophys. 1995, 33, 163-198.

11. Gupta, A.C.; Banerjee, D.P.K.; Ashok, N.M.; Joshi, U.C. Near infrared intraday variability of Mrk 421. Astron. Astrophys. 2004, 422, 505-508.

12. Miller, H.R.; Carini, M.T.; Goodrich, B.D. Detection of microvariability for BL Lacertae objects. Nature 1989, 337, 627-629.

13. Carini, M.T.; Miller, H.R.; Goodrich, B.D. The timescales of the optical variability of blazars. I-OQ 530. Astron. J. 1990, 100, 347-355.

14. Carini, M.T.; Miller, H.R.; Noble, J.C.; Sadun, A.C. The timescales of the optical variability of blazars. II-AP Librae. Astron. J. 1991, 101, 1196-1201.

15. Carini, M.T.; Miller, H.R.; Noble, J.C.; Goodrich, B.D. The timescales of the optical variability of blazars. III-OJ 287 and BL Lacertae. Astron. J. 1992, 104, 15-27.

16. Carini, M.T.; Miller, H.R. The optical variability of PKS 2155-304. Astrophys. J. 1992, 385, 146-150.

17. Quirrenbach, A.; Witzel, A.; Wagner, S.; Sanchez-Pons, F.; Krichbaum, T.P.; Wegner, R.; Anton, K.; Erkens, U.; Haehnelt, M.; Zensus, J.A.; et al. Correlated radio and optical variability in the BL Lacertae object 0716+714. Astrophys. J. 1991, 372, L71-L74.

18. Heidt, J.; Wagner, S.J. Statistics of optical intraday variability in a complete sample of radio-selected BL Lacertae objects. Astron. Astrophys. 1996, 305, 42-52.

19. Heidt, J.; Wagner, S.J. Intraday variability in X-ray selected BL Lacertae objects. Astron. Astrophys. 1998, 329, 853-862.

20. Sagar, R.; Gopal-Krishna; Mohan, V.; Pandey, A.K.; Bhatt, B.C.; Wagner, S.J. Multi-colour optical monitoring of the intra-day variable blazar S5 0716+71. Astron. Astrophys. Supp. 1999, 134, 453-461.

21. Montagni, F.; Maselli, A.; Massaro, E.; Nesci, R.; Sclavi, S.; Maesano, M. The intra-night optical variability of the bright BL Lacertae object S5 0716+714. Astron. Astrophys. 2006, 451, 435-442.

22. Aharonian, F.; Akhperjanian, A.G.; Bazer-Bachi, A.R.; Behera, B.; Beilicke, M.; Benbow, W.; Berge, D.; Bernlöhr, K.; Boisson, C.; Bolz, O.; et al. An Exceptional Very High Energy Gamma-Ray Flare of PKS 2155-304. Astrophys. J. 2007, 664, L71-L74.

23. Gupta, A.C.; Fan, J.H.; Bai, J.M.; Wagner, S.J. Optical Intra-Day Variability in Blazars. Astron. J. 2008, 135, 1384-1394.

24. Gupta, A.C.; Cha, S.-M.; Lee, S.; Jin, H.; Pak, S.; Cho, S.-H.; Moon, B.; Park, Y.; Yuk, I.-S.; Nam, U.-W.; et al. Multicolor Near-Infrared Intra-Day and Short-Term Variability of the Blazar S5 0716+714. Astron. J. 2008, 136, 2359-2366.

25. Gupta, A.C.; Krichbaum, T.P.; Wiita, P.J.; Rani, B.; Sokolovsky, K.V.; Mohan, P.; Mangalam, A.; Marchili, N.; Fuhrmann, L.; Agudo, I.; et al. Multiwavelength intraday variability of the BL Lacertae S5 0716+714. Mon. Not. R. Astron. Soc. 2012, 425, 1357-1370.

26. Gupta, A.C.; Agarwal, A.; Bhagwan, J.; Strigachev, A.; Bachev, R.; Semkov, E.; Gaur, H.; Damljanovic, G.; Vince, O.; Wiita, P.J. Multiband optical variability of three TeV blazars on diverse timescales. Mon. Not. R. Astron. Soc. 2016, 458, 1127-1137.

27. Gupta, A.C.; Agarwal, A.; Mishra, A.; Gaur, H.; Wiita, P.J.; Gu, M.F.; Kurtanidze, O.M.; Damljanovic, G.; Uemura, M.; Semkov, E.; et al. Multiband optical variability of the blazar OJ 287 during its outbursts in 2015-2016. Mon. Not. R. Astron. Soc. 2017, 465, 4423-4433. 
28. Gaur, H.; Gupta, A.C.; Lachowicz, P.; Wiita, P.J. Detection of Intra-day Variability Timescales of Four High-energy Peaked Blazars with XMM-Newton. Astrophys. J. 2010, 718, 279-291.

29. Gaur, H.; Gupta, A.C.; Wiita, P.J. Multiwavelength Variability of the Blazars Mrk 421 and 3C 454.3 in the High State. Astron. J. 2012, 143, 23.

30. Gaur, H.; Gupta, A.C.; Strigachev, A.; Bachev, R.; Semkov, E.; Wiita, P.J.; Peneva, S.; Boeva, S.; Kacharov, N.; Mihov, B.; et al. Quasi-simultaneous two-band optical variability of the blazars 1ES 1959+650 and 1ES 2344+514. Mon. Not. R. Astron. Soc. 2012, 420,3147-3162.

31. Gaur, H.; Gupta, A.C.; Strigachev, A.; Bachev, R.; Semkov, E.; Wiita, P.J.; Peneva, S.; Boeva, S.; Slavcheva-Mihova, L.; Mihov, B.; et al. Optical flux and spectral variability of blazars. Mon. Not. R. Astron. Soc. 2012, 425, 3002-3023.

32. Gaur, H.; Gupta, A.C.; Bachev, R.; Strigachev, A.; Semkov, E.; Böttcher, M.; Wiita, P.J.; de Diego, J.A.; Gu, M.F.; Guo, H.; et al. Nature of intranight optical variability of BL Lacertae. Mon. Not. R. Astron. Soc. 2015, 452, 4263-4273.

33. Foschini, L.; Ghisellini, G.; Tavecchio, F.; Bonnoli, G.; Stamerra, A. Search for the shortest variability at gamma rays in flat-spectrum radio quasars. Astron. Astrophys. 2011, 530, A77.

34. Kushwaha, P.; Singh, K.P.; Sahayanathan, S. Brightest Fermi-LAT Flares of PKS 1222+216: Implications on Emission and Acceleration Processes. Astrophys. J. 2014, 796, 61.

35. Paliya, V.S.; Sahayanathan, S.; Stalin, C.S. Multi-Wavelength Observations of 3C 279 During the Extremely Bright Gamma-Ray Flare in 2014 March-April. Astrophys. J. 2015, 803, 15.

36. Paliya, V.S. Fermi-Large Area Telescope Observations of the Exceptional Gamma-Ray Flare from 3C 279 in 2015 June. Astrophys. J. 2015, 808, L48.

37. Kalita, N.; Gupta, A.C.; Wiita, P.J.; Bhagwan, J.; Duorah, K. Multiband variability in the blazar 3C 273 with XMM-Newton. Mon. Not. R. Astron. Soc. 2015, 451, 1356-1365.

38. Agarwal, A.; Gupta, A.C. Multiband optical variability studies of BL Lacertae. Mon. Not. R. Astron. Soc. 2015, 450, 514-551.

39. Agarwal, A.; Gupta, A.C.; Bachev, R.; Strigachev, A.; Semkov, E.; Wiita, P.J.; Böttcher, M.; Boeva, S.; Gaur, H.; $\mathrm{Gu}$, M.F.; et al. Multiband optical-NIR variability of blazars on diverse timescales. Mon. Not. R. Astron. Soc. 2015, 451, 3882-3897.

40. Agarwal, A.; Gupta, A.C.; Bachev, R.; Strigachev, A.; Semkov, E.; Wiita, P.J.; Fan, J.H.; Pandey, U.S.; Boeva, S.; Spassov, B. Multiband optical variability of the blazar S5 0716+714 in outburst state during 2014-2015. Mon. Not. R. Astron. Soc. 2016, 455, 680-690.

41. Ackermann, M.; Anantua, R.; Asano, K.; Baldini, L.; Barbiellini, G.; Bastieri, D.; Becerra Gonzalez, J.; Bellazzini, R.; Bissaldi, E.; Blandford, R.D.; et al. Minute-timescale $>100 \mathrm{MeV} \gamma$-ray Variability during the Giant Outburst of Quasar 3C 279 Observed by Fermi-LAT in 2015 June. Astrophys. J. 2016, 824, L20.

42. Pandey, A.; Gupta, A.C.; Wiita, P.J. X-ray Intraday Variability of Five TeV Blazars with NuSTAR. Astrophys. J. 2017, 841, 123.

43. Aggrawal, V.; Gupta, A.C.; Zhang, Z.; Wiita, P.J.; Yadav, K.K.; Tiwari, S.N. X-ray Intraday Variability of the TeV Blazar Mrk 421 with Chandra. Mon. Not. R. Astron. Soc. 2017, submitted.

44. Romero, G.E.; Cellone, S.A.; Combi, J.A. Optical microvariability of southern AGNs. Astron. Astrophys. Suppl. 1999, 135, 477-486.

45. Jang, M.; Miller, H.R. The Microvariability of Selected Radio-Quiet and Radio-Loud QSOs. Astron. J. 1997, 114, 565-574.

46. Stalin, C.S.; Gopal-Krishna; Sagar, R.; Wiita, P.J. Intranight optical variability of radio-quiet and radio lobe-dominated quasars. Mon. Not. R. Astron. Soc. 2004, 350, 175-188.

47. de Diego, J.A. Testing Tests on Active Galactic Nucleus Microvariability. Astron. J. 2010, 139, $1269-1282$.

48. de Diego, J.A. On the Reliability of Microvariability Tests in Quasars. Astron. J. 2014, 148, 93.

49. de Diego, J.A.; Polednikova, J.; Bongiovanni, A.; Pérez García, A.M.; De Leo, M.A.; Verdugo, T.; Cepa, J. Testing Microvariability in Quasar Differential Light Curves Using Several Field Stars. Astron. J. 2015, 150,44 .

50. de Diego, J.A. Dultzin-Hacyan, D.; Ramírez, A.; Benítez, E. A Comparative Study of the Microvariability Properties in Radio-loud and Radio-quiet Quasars. Astrophys. J. 1998, 501, 69-81. 
51. Edelson, R.; Turner, T.J.; Pounds, K.; Vaughan, S.; Markowitz, A.; Marshall, H.; Dobbie, P.; Warwick, R. X-ray Spectral Variability and Rapid Variability of the Soft X-ray Spectrum Seyfert 1 Galaxies Arakelian 564 and Ton S180. Astrophys. J. 2002, 568, 610-626.

52. Vaughan, S.; Edelson, R.; Warwick, R.S.; Uttley, P. On characterizing the variability properties of X-ray light curves from active galaxies. Mon. Not. R. Astron. Soc. 2003, 345, 1271-1284.

53. Bhagwan, J.; Gupta, A.C.; Papadakis, I.E.; Wiita, P.J. Flux and spectral variability of the blazar PKS 2155-304 with XMM-Newton: Evidence of particle acceleration and synchrotron cooling. New Astron. 2016, 44, $21-28$.

54. Gupta, A.C.; Kalita, N.; Gaur, H.; Duorah, K. Peak of spectral energy distribution plays an important role in intra-day variability of blazars? Mon. Not. R. Astron. Soc. 2016, 462, 1508-1516.

55. Cohen, R.D.; Smith, H.E.; Junkkarinen, V.T.; Burbidge, E.M. The nature of the BL Lacertae object AO 0235+164. Astrophys. J. 1987, 318, 577-584.

56. Nilsson, K.; Charles, P.A.; Pursimo, T.; Takalo, L.O.; Sillanpä̈̈, A.; Teerikorpi, P. The complex environment of AO 0235+164. Astron. Astrophys. 1996, 314, 754-762.

57. Marscher A.P.; Gear, W.K. Models for high-frequency radio outbursts in extragalactic sources, with application to the early 1983 millimeter-to-infrared flare of 3C 273. Astrophys. J. 1985, 298, 114-127.

58. Qian, S.J.; Quirrenbach, A.; Witzel, A.; Krichbaum, T.P.; Hummel, C.A.; Zensus, J.A. A model for the rapid radio variability in the quasar $0917+624$. Astron. Astrophys. 1991, 241, 15-21.

59. Marscher, A.P.; Gear, W.K.; Travis, J.P. Variability of Nonthermal Continuum Emission in Blazars. In Variability of Blazars; Valtaoja, E., Valtonen, M., Eds.; Cambridge University Press: Cambridge, UK, 1992; p. 85.

60. Kato S.; Fukue, J. Trapped Radial Oscillations of Gaseous Disks around a Black Hole. Pub. Astron. Soc. Jpn. 1980, 32, 377-388.

61. Nowak, M.A.; Wagoner, R.V. Diskoseismology: Probing accretion disks. II-G-modes, gravitational radiation reaction, and viscosity. Astrophys. J. 1992, 393, 697-707.

62. Zhang, X.-H.; Bao, G. The rotation of accretion disks and the power spectra of X-ray 'flickering'. Astron. Astrophys. 1991, 246, 21-31.

63. Mangalam, A.; Wiita, P.J. Accretion disk models for optical and ultraviolet microvariability in active galactic nuclei. Astrophys. J. 1993, 406, 420-429.

64. Sol, H.; Pelletier, G.; Asseo, E. Two-flow model for extragalactic radio jets. Mon. Not. R. Astron. Soc. 1989, $237,411-429$.

65. Atwood, W.B.; Abdo, A.A.; Ackermann, M.; Althouse, W.; Anderson, B.; Axelsson, M.; Baldini, L.; Ballet, J.; Band, D.L.; Barbiellini, G.; et al. The Large Area Telescope on the Fermi Gamma-Ray Space Telescope Mission. Astrophys. J. 2009, 697, 1071-1102.

66. Brown, A.M. Locating the $\gamma$-ray emission region of the flat spectrum radio quasar PKS 1510-089. Mon. Not. R. Astron. Soc. 2013, 431, 824-835.

67. Paliya, V.S.; Diltz, C.; Böttcher, M.; Stalin, C.S.; Buckley, D. A Hard Gamma-Ray Flare from 3C 279 in 2013 December. Astrophys. J. 2016, 817, 61.

68. Hayashida, M.; Nalewajko, K.; Madejski, G.M.; Sikora, M.; Itoh, R.; Ajello, M.; Blandford, R.D.; Buson, S.; Chiang, J.; Fukazawa, Y.; et al. Rapid Variability of Blazar 3C 279 during Flaring States in 2013-2014 with Joint Fermi-LAT, NuSTAR, Swift, and Ground-Based Multiwavelength Observations. Astrophys. J. 2015, 807, 79.

69. González-Martín, O.; Vaughan, S. X-ray variability of 104 active galactic nuclei. XMM-Newton power-spectrum density profiles. Astron. Astrophys. 2012, 544, A80.

70. Gupta, A.C.; Joshi, U.C. Intra-night optical variability of luminous radio-quiet QSOs. Astron. Astrophys. 2005, 440, 855-865.

71. Bai, J.M.; Xie, G.Z.; Li, K.H.; Zhang, X.; Liu, W.W. The intraday variability in the radio-selected and X-ray-selected BL Lacertae objects. Astron. Astrophys. Supp. 1998, 132, 83-92.

72. Bai, J.M.; Xie, G.Z.; Li, K.H.; Zhang, X.; Liu, W.W. Photometric monitoring of three BL Lacertae objects in 1993-1998. Astron. Astrophys. Supp. 1999, 136, 455-460.

73. Dai, B.Z.; Xie, G.Z.; Li, K.H.; Zhou, S.B.; Liu, W.W.; Jiang, Z.J. Rapid Optical Variability of Gamma-Ray-loud Blazars. Astron. J. 2001, 122, 2901-2912.

74. Xie, G. Z.; Li, K. H.; Zhang, X.; Bai, J. M.; Liu, W. W. Optical Monitoring Sample of the GEV Gamma-Ray-loud Blazars. Astrophys. J. 1999, 522, 846-862. 
75. Xie, G.Z.; Li, K.H.; Bai, J.M.; Dai, B.Z.; Liu, W.W.; Zhang, X.; Xing, S.Y. Search for Short Variability Timescale of the GEV Gamma-Ray-Loud Blazars. Astrophys. J. 2001, 548, 200-212.

76. Xie, G.Z.; Zhou, S.B.; Dai, B.Z.; Liang, E.W.; Li, K. H.; Bai, J.M.; Xing, S.Y.; Liu, W.W. Photometric monitoring of 12 BL Lacertae objects. Mon. Not. R. Astron. Soc. 2002, 329, 689-699.

77. Xie, G.Z.; Zhou, S.B.; Li, K.H.; Dai, H.; Chen, L.E.; Ma, L. CCD photometric studies of rapid variability in eight blazars. Mon. Not. R. Astron. Soc. 2004, 348, 831-845.

78. Fan, J.H.; Qian, B.C.; Tao, J. Optical observations of BL Lacertae from 1997 to 1999. Astron. Astrophys. 2001, $369,758-762$.

79. Fan, J.H.; Kurtanidze, O.M.; Nikolashvili, M.G.; Gupta, A.C.; Zhang, J.-S.; Yuan, Y.-H. Optical Photometric Observations of $\gamma$-Ray Loud Blazars. Chin. J. Astron. Astrophy., 2004, 4, 133-142.

80. Qian, B.; Tao, J. Optical Monitoring of PKS 0735+178 from 1995 to 2001 and Its Historical Periodic Light Curve. Pub. Astron. Soc. Pacific 2004, 116, 161-169.

81. Wu, J.; Peng, B.; Zhou, X.; Ma, J.; Jiang, Z.; Chen, J. Optical Monitoring of BL Lacertae Object S5 0716+714 with High Temporal Resolution. Astron. J. 2005, 129, 1818-1826.

82. Wu, J.; Zhou, X.; Ma, J.; Wu, Z.; Jiang, Z.; Chen, J. Optical Monitoring of BL Lacertae Object S5 0716+714 with a Novel Multipeak Interference Filter. Astron. J. 2007, 133, 1599-1608.

83. Poon, H.; Fan, J.H.; Fu, J.N. The Optical Microvariability and Spectral Changes of the BL Lacertae Object S5 0716+714. Astrophys. J. Suppl. 2009, 185, 511-525.

84. Hu, S.M.; Chen, X.; Guo, D.F.; Jiang, Y.G.; Li, K. Quasi-simultaneous multicolour optical variability of S5 0716+714. Mon. Not. R. Astron. Soc. 2014, 443, 2940-2949.

85. Xiong, D.; Zhang, H.; Zhang, X.; Yi, T.; Bai, J.; Wang, F.; Liu, H.; Zheng, Y. Multi-color Optical Monitoring of MRK 501 from 2010 to 2015. Astrophys. J. Suppl. 2016, 222, 24.

86. Li, Y.T.; Hu, S.M.; Jiang, Y.G.; Chen, X.; Priyadarshi, S.; Li, K.; Guo, Y.C.; Guo, D. Symmetry Analysis of the Multi-band Optical Variability of BL LAC S5 0716+714 in Intranight and Longer Timescales. Pub. Astron. Soc. Jpn. 2017, 129, 014101.

87. Feng, H.-C.; Liu, H.T.; Fan, X.L.; Zhao, Y.; Bai, J.M.; Wang, F.; Xiong, D.R.; Li, S.K. Search for Intra-day Optical Variability in Mrk 501. Astrophys. J. 2017, 849, 161.

88. Hong, S.; Xiong, D.; Bai, J. Multicolor Optical Monitoring of the BL Lacertae Object S5 0716+714 during the 2012 Outburst. Astron. J. 2017, 154, 42.

89. Ghosh, K.K.; Ramsey, B.D.; Sadun, A.C.; Soundararajaperumal, S. Optical Variability of Blazars. Astrophys. J. Suppl. 2000, 127, 11-26.

90. Ghosh, K.K.; Kim, C.; Ramsey, B.D.; Soundararajaperumal, S. Optical Microvariability of Blazars. J. Korean Astron. Soc. 2001, 34, 9-15.

91. Stalin, C.S.; Gupta, A.C.; Gopal-Krishna; Wiita, P.J.; Sagar, R. Intranight optical variability of BL Lacs, radio-quiet quasars and radio-loud quasars. Mon. Not. R. Astron. Soc. 2005, 356, 607-614.

92. Stalin, C.S.; Gopal-Krishna; Sagar, R.; Wiita, P.J.; Mohan, V.; Pandey, A.K. Multiband optical monitoring of the blazars S5 0716+714 and BL Lacertae. Mon. Not. R. Astron. Soc. 2006, 366, 1337-1345.

93. Goyal, A.; Gopal-Krishna; Anupama, G.C.; Sahu, D.K.; Sagar, R.; Britzen, S.; Karouzos, M.; Aller, M.F.; Aller, H.D. Unusual optical quiescence of the classical BL Lac object PKS 0735+178 on intranight timescale. Mon. Not. R. Astron. Soc. 2009, 399, 1622-1632.

94. Rani, B.; Gupta, A.C.; Joshi, U.C.; Ganesh, S.; Wiita, P.J. Optical intraday variability studies of 10 low energy peaked blazars. Mon. Not. R. Astron. Soc. 2011, 413, 2157-2172.

95. Villata, M.; Mattox, J.R.; Massaro, E.; Nesci, R.; Catalano, S.; Frasca, A.; Raiteri, C.M.; Sobrito, G.; Tosti, G.; Nucciarelli, G.; et al. The 0716+714 WEBT campaign of February 1999. Astron. Astrophys. 2000, 363, 108-116.

96. Villata, M.; Raiteri, C.M.; Kurtanidze, O.M.; Nikolashvili, M.G.; Ibrahimov, M.A.; Papadakis, I.E.; Tsinganos, K.; Sadakane, K.; Okada, N.; Takalo, L.O.; et al. The WEBT BL Lacertae Campaign 2000. Astron. Astrophys. 2002, 390, 407-421.

97. Villata, M.; Raiteri, C.M.; Kurtanidze, O.M.; Nikolashvili, M.G.; Ibrahimov, M.A.; Papadakis, I.E.; Tosti, G.; Hroch, F.; Takalo, L.O.; Sillanpää, A. et al. The WEBT BL Lacertae Campaign 2001 and its extension. Optical light curves and colour analysis 1994-2002. Astron. Astrophys. 2004, 421, 103-114.

98. Villata, M.; Raiteri, C.M.; Larionov, V.M.; Kurtanidze, O.M.; Nilsson, K.; Aller, M.F.; Tornikoski, M.; Volvach, A.; Aller, H.D.; Arkharov, A.A.; et al. Multifrequency monitoring of the blazar 0716+714 during the GASP-WEBT-AGILE campaign of 2007. Astron. Astrophys. 2008, 481, L79-L82. 
99. Romero, G.E.; Cellone, S.A.; Combi, J.A.; Andruchow, I. Optical microvariability of EGRET blazars. Astron. Astrophys. 2002, 390, 431-438.

100. Papadakis, I.E.; Boumis, P.; Samaritakis, V.; Papamastorakis, J. Multi-band optical micro-variability observations of BL Lacertae. Astron. Astrophys. 2003, 397, 565-573.

101. Papadakis, I.E.; Samaritakis, V.; Boumis, P.; Papamastorakis, J. Multi-band optical micro-variability observations of the BL Lac object S4 0954+658. Astron. Astrophys. 2004, 426, 437-443.

102. Ostorero, L.; Wagner, S.J.; Gracia, J.; Ferrero, E.; Krichbaum, T.P.; Britzen, S.; Witzel, A.; Nilsson, K.; Villata, M.; Bach, U.; et al. Testing the inverse-Compton catastrophe scenario in the intra-day variable blazar S5 0716+71. I. Simultaneous broadband observations during November 2003. Astron. Astrophys. 2006, 451, 797-807.

103. Xilouris, E.M.; Papadakis, I.E.; Boumis, P.; Dapergolas, A.; Alikakos, J.; Papamastorakis, J.; Smith, N.; Goudis, C.D. B and I-band optical micro-variability observations of the BL Lac objects S5 2007+777 and 3C 371. Astron. Astrophys. 2006, 448, 143-153.

104. Bachev, R.; Semkov, E.; Strigachev, A.; Mihov, B.; Gupta, A.C.; Peneva, S.; Ovcharov, E.; Valcheva, A.; Lalova, A. Intranight variability of 3C 454.3 during its 2010 November outburst. Astron. Astrophys. 2011, 528, L10.

105. Bachev, R.; Semkov, E.; Strigachev, A.; Gupta, A.C.; Gaur, H.; Mihov, B.; Boeva, S.; Slavcheva-Mihova, L. The nature of the intra-night optical variability in blazars. Mon. Not. R. Astron. Soc. 2012, 424, 2625-2634.

106. Bachev, R. Violent intranight optical variability of the blazar S4 0954+65 during its unprecedented 2015 February outburst. Mon. Not. R. Astron. Soc. 2015, 451, L21-L24.

107. Bachev, R.; Popov, V.; Strigachev, A.; Semkov, E.; Ibryamov, S.; Spassov, B.; Latev, G.; Muñoz Dimitrova, R.V.; Boeva, S. Intra-night variability of the blazar CTA 102 during its 2012 and 2016 giant outbursts. Mon. Not. R. Astron. Soc. 2017, 471, 2216-2223.

108. Bhatta, G.; Webb, J.R.; Hollingsworth, H.; Dhalla, S.; Khanuja, A.; Bachev, R.; Blinov, D.A.; Böttcher, M.; Bravo Calle, O.J.A.; Calcidese, P.; et al. The 72-h WEBT microvariability observation of blazar S5 0716+714 in 2009. Astron. Astrophys. 2013, 558, A92.

109. Bhatta, G.; Stawarz, Ł.; Ostrowski, M.; Markowitz, A.; Akitaya, H.; Arkharov, A.A.; Bachev, R.; Benítez, E.; Borman, G.A.; Carosati, D.; et al. Multifrequency Photo-polarimetric WEBT Observation Campaign on the Blazar S5 0716+714: Source Microvariability and Search for Characteristic Timescales. Astrophys. J. 2016, $831,92$.

110. Gupta, S.P.; Pandey, U.S.; Singh, K.; Rani, B.; Pan, J.; Fan, J.H.; Gupta, A.C. Optical intra-day variability timescales and black hole mass of the blazars. New Astron. 2012, 17, 8-17.

111. Wagner, S.; Sanchez-Pons, F.; Quirrenbach, A.; Witzel, A. Simultaneous optical and radio monitoring of rapid variability in quasars and BL Lac objects. Astron. Astrophys. 1990, 235, L1-L4.

112. Agudo, I.; Krichbaum, T.P.; Ungerechts, H.; Kraus, A.; Witzel, A.; Angelakis, E.; Fuhrmann, L.; Bach, U.; Britzen, S.; Zensus, J.A.; et al. Testing the inverse-Compton catastrophe scenario in the intra-day variable blazar S5 $0716+71$. II. A search for intra-day variability at millimetre wavelengths with the IRAM $30 \mathrm{~m}$ telescope. Astron. Astrophys. 2006, 456, 117-129.

113. Gabányi, K.É.; Marchili, N.; Krichbaum, T.P.; Britzen, S.; Fuhrmann, L.; Witzel, A.; Zensus, J.A.; Müller, P.; Liu, X.; Song, H.G.; et al. The IDV source J 1128+5925, a new candidate for annual modulation? Astron. Astrophys. 2007, 470, 83-95.

114. Gabányi, K.É.; Marchili, N.; Krichbaum, T.P.; Fuhrmann, L.; Müller, P.; Zensus, J.A.; Liu, X.; Song, H.G. Very Long Baseline Array observations of the intraday variable source J1128+592. Astron. Astrophys. 2009, 508, 161-171.

115. Fuhrmann, L.; Krichbaum, T.P.; Witzel, A.; Kraus, A.; Britzen, S.; Bernhart, S.; Impellizzeri, C.M.V.; Agudo, I.; Villata, M.; Bach, U.; et al. Testing the inverse-Compton catastrophe scenario in the intra-day variable blazar S5 0716+71. III. Rapid and correlated flux density variability from radio to sub-mm bands. Astron. Astrophys. 2008, 490, 1019-1037.

116. Marchili, N.; Krichbaum, T.P.; Liu, X.; Song, H.-G.; Anderson, J.M.; Witzel, A.; Zensus, J.A. On the influence of the Sun on the rapid variability of compact extragalactic sources. Astron. Astrophys. 2011, 530, A129.

117. Marchili, N.; Krichbaum, T.P.; Liu, X.; Song, H.-G.; Gabányi, K.É.; Fuhrmann, L.; Witzel, A.; Zensus, J.A. A seasonal cycle and an abrupt change in the variability characteristics of the intraday variable source S4 0954+65. Astron. Astrophys. 2012, 542, A121. 
118. Liu, B.-R.; Liu, X.; Marchili, N.; Liu, J.; Mi, L.-G.; Krichbaum, T.P.; Fuhrmann, L.; Zensus, J.A. Two-year monitoring of intra-day variability of quasar $1156+295$ at $4.8 \mathrm{GHz}$. Astron. Astrophys. 2013, 555, A134.

119. Liu, X.; Song, H.-G.; Marchili, N.; Liu, B.-R.; Liu, J.; Krichbaum, T.P.; Fuhrmann, L.; Zensus, J.A. Intra-day variability observations of S5 0716+714 over 4.5 years at $4.8 \mathrm{GHz}$. Astron. Astrophys. 2012, 543, A78.

120. Liu, X.; Mi, L.-G.; Liu, J.; Cui, L.; Song, H.-G.; Krichbaum, T.P.; Kraus, A.; Fuhrmann, L.; Marchili, N.; Zensus, J.A. Intra-day variability observations and the VLBI structure analysis of quasar S4 0917+624. Astron. Astrophys. 2015, 578, A34.

121. Liu, X.; Yang, P.P.; Liu, J.; Liu, B.R.; Hu, S.M.; Kurtanidze, O.M.; Zola, S.; Kraus, A.; Krichbaum, T.P.; $\mathrm{Su}, \mathrm{R} . Z$; et al. Radio and optical intra-day variability observations of five blazars. Mon. Not. R. Astron. Soc. 2017, 469, 2457-2463.

122. Gierliński, M.; Middleton, M.; Ward, M.; Done, C. A periodicity of $\sim 1 \mathrm{~h}$ in X-ray emission from the active galaxy RE J1034+396. Nature 2008, 455, 369-371.

123. Espaillat, C.; Bregman, J.; Hughes, P.; Lloyd-Davies, E. Wavelet Analysis of AGN X-ray Time Series: A QPO in 3C 273? Astrophys. J. 2008, 679, 182-193.

124. Gupta, A.C.; Srivastava, A.K.; Wiita, P.J. Periodic Oscillations in the Intra-Day Optical Light Curves of the Blazar S5 0716+714. Astrophys. J. 2009, 690, 216-223.

125. Lachowicz, P.; Gupta, A.C.; Gaur, H.; Wiita, P.J. A 4.6 h quasi-periodic oscillation in the BL Lacertae PKS 2155-304? Astron. Astrophys. 2009, 506, L17-L20.

126. Rani, B.; Wiita, P.J.; Gupta, A.C. Nearly Periodic Fluctuations in the Long-term X-ray Light Curves of the Blazars AO 0235+164 and 1ES 2321+419. Astrophys. J. 2009, 696, 2170-2178.

127. Rani, B.; Gupta, A.C.; Joshi, U.C.; Ganesh, S.; Wiita, P.J. Quasi-periodic Oscillations of 15 Minutes in the Optical Light Curve of the BL Lac S5 0716+714. Astrophys. J. 2010, 719, L153-L157.

128. Lin, D.; Irwin, J.A.; Godet, O.; Webb, N.A.; Barret, D. A 3.8 h Periodicity from an Ultrasoft Active Galactic Nucleus Candidate. Astrophys. J. 2013, 776, L10.

129. Sandrinelli, A.; Covino, S.; Treves, A. Long and short term variability of seven blazars in six near-infrared/ optical bands. Astron. Astrophys. 2014, 562, A79.

130. Sandrinelli, A.; Covino, S.; Dotti, M.; Treves, A. Quasi-periodicities at Year-like Timescales in Blazars. Astron. J. 2016, 151, 54.

131. Sandrinelli, A.; Covino, S.; Treves, A. Gamma-Ray and Optical Oscillations in PKS 0537-441. Astrophys. J. 2016, 820, 20.

132. Sandrinelli, A.; Covino, S.; Treves, A.; Lindfors, E.; Raiteri, C.M.; Nilsson, K.; Takalo, L.O.; Reinthal, R.; Berdyugin, A.; Ramazani, V.F.; et al. Gamma-ray and optical oscillations of 0716+714, MRK 421, and BL Lacertae. Astron. Astrophys. 2017, 600, A132.

133. Graham, M.J.; Djorgovski, S.G.; Stern, D.; Glikman, E.; Drake, A.J.; Mahabal, A.A.; Donalek, C.; Larson, S.; Christensen, E. A possible close supermassive black-hole binary in a quasar with optical periodicity. Nature 2015, 518, 74-76.

134. Ackermann, M.; Ajello, M.; Albert, A.; Atwood, W.B.; Baldini, L.; Ballet, J.; Barbiellini, G.; Bastieri, D.; Becerra Gonzalez, J.; Bellazzini, R.; et al. Multiwavelength Evidence for Quasi-periodic Modulation in the Gamma-Ray Blazar PG 1553+113. Astrophys. J. 2015, 813, L41.

135. Alston, W.N.; Markevičiūtè, J.; Kara, E.; Fabian, A.C.; Middleton, M. Detection of a QPO in five XMM-Newton observations of RE J1034+396. Mon. Not. R. Astron. Soc. 2014, 445, L16-L20.

136. Alston, W.N.; Parker, M.L.; Markevičiūtè, J.; Fabian, A.C.; Middleton, M.; Lohfink, A.; Kara, E.; Pinto, C. Discovery of an $\sim 2$-h high-frequency $\mathrm{X}$-ray QPO and iron $\mathrm{K}_{\alpha}$ reverberation in the active galaxy MS 2254.9-3712. Mon. Not. R. Astron. Soc. 2015, 449, 467-476.

137. Pan, H.-W.; Yuan, W.; Yao, S.; Zhou, X.-L.; Liu, B.; Zhou, H.; Zhang, S.-N. Detection of a Possible X-ray Quasi-periodic Oscillation in the Active Galactic Nucleus 1H 0707-495. Astrophys. J. 2016, 819, L19.

138. Bhatta, G.; Zola, S.; Stawarz, Ł.; Ostrowski, M.; Winiarski, M.; Ogłoza, W.; Dróżdz, M.; Siwak, M.; Liakos, A.; Kozieł-Wierzbowska, D.; et al. Detection of Possible Quasi-periodic Oscillations in the Long-term Optical Light Curve of the BL Lac Object OJ 287. Astrophys. J. 2016, 832, 47.

139. Bhatta, G. Radio and $\gamma$-ray Variability in the BL Lac PKS 0219-164: Detection of Quasi-periodic Oscillations in the Radio Light Curve. Astrophys. J. 2017, 847, 7.

140. Chakrabarti, S.K.; Wiita, P.J. Spiral shocks in accretion disks as a contributor to variability in active galactic nuclei. Astrophys. J. 1993, 411, 602-609. 
141. Abramowicz, M.A. QPO as the Rosetta Stone for understanding black hole accretion. Astron. Nachrichten 2005, 326, 782-786.

142. Perez, C.A.; Silbergleit, A.S.; Wagoner, R.V.; Lehr, D.E. Relativistic Diskoseismology. I. Analytical Results for “Gravity Modes". Astrophys. J. 1997, 476, 589-604.

143. Camenzind, M.; Krockenberger, M. The lighthouse effect of relativistic jets in blazars-A geometric origin of intraday variability. Astron. Astrophys. 1992, 255, 59-62.

144. Gopal-Krishna; Wiita, P.J. Swinging jets and the variability of active galactic nuclei. Astron. Astrophys. 1992, 259, 109-117.

(C) 2017 by the author. Licensee MDPI, Basel, Switzerland. This article is an open access article distributed under the terms and conditions of the Creative Commons Attribution (CC BY) license (http:// creativecommons.org/licenses/by/4.0/). 